\title{
Effectiveness of Kinesio Taping and Retro Walking Exercise on the Knee Range of Motion in Older People with Osteoarthritis
}

\author{
Yulian Pristanti Sani Putri ${ }^{1 *}$, Atika Yulianti ${ }^{1}$
}

\section{ABSTRACT}

Background: Osteoarthritis $(O A)$ is a degenerative disease of the synovial joint marked by chondropathy with the proliferation of new bone and also renovation of joint contour. Knee $0 \mathrm{~A}$ is associated with age, gender, and excessive weight conditions, conservatively treated using physical therapy intervention such as Kinesio taping and retro walking exercise to enhance the range of motion of knee joint. This study aimed to compare the effectiveness of Kinesio taping and retro walking exercise on the knee range of motion in older people with osteoarthritis.

Methods: This study used quasi-experimental two groups pretest and post-test design, which was involving 30 participants by using purposive sampling as a sample collection technique. Additionally, the sample was divided into two intervention groups of 14 participants in Kinesio tape group and 16 participants in retro walking exercise group. Furthermore, the obtained data were analyzed using the Wilcoxon sign ranked test and Mann Whitney $U$ test.

Results: After the intervention period, pre-test and post-test within-group analysis showed a significant improvement of knee ROM in the Kinesio taping group $(P<0.001)$ and retro walking group $(P=0.019)$. Furthermore, the analysis for mean differences of pre-test and post-test between-group comparison accepted the study hypothesis that Kinesio taping had a bigger effect than retro walking exercise to improve knee ROM ( $P=0.010)$.

Conclusion: Both Kinesio taping and retro walking exercises were influencing ROM improvement in the elderly. However, the mean result showed that Kinesio taping was giving more influence in enhancing ROM as compared to retro walking exercise.

Keywords: elderly, Kinesio taping, knee osteoarthritis, retro walking exercise

Cite this Article: Putri, Y.P.S., Yulianti, A. 2020. Effectiveness of Kinesio Taping and Retro Walking Exercise on the Knee Range of Motion in Older People with 0steoarthritis. Physical Therapy Journal of Indonesia 1(2): 23-25.

'Physiotherapy Department, Universitas Muhammadiyah Malang
*Corresponding author: Yulian Pristanti Sani Putri, Physiotherapy Department, Universitas Muhammadiyah Malang; ntypsp@gmail.com
Received : 2020-06-30 Accepted : 2020-10-30 Published : 2020-12-01

\section{INTRODUCTION}

Osteoarthritis (OA) is a degenerative disease of the synovial joint marked by chondropathy with the proliferation of new bone and also renovation of joint contour, the knee $\mathrm{OA}$ is one of the most common OAs in older people that associated with disability. ${ }^{1}$ According to the World Health Organization, the range age of older people is started from sixty years old and above. ${ }^{2}$ Several symptoms that usually bother the older with knee OA including joint pain and stiffness, swelling, crackling, muscle weakness, and poor knee specific quality of life. ${ }^{3}$

The knee range of motion (ROM) refers to the degree of movement that the knee joint can move in a certain range. Normal knee ROM is about $130^{\circ}$ and $0^{\circ}$ for flexion and extension, respectively. ${ }^{4}$ The goniometer is one of the common, reliable, and valid tools to use either actively or passively to measure the knee ROM. ${ }^{5}$

Nowadays, to improve the joint stiffness of knee OA, non-pharmacological therapies were suggested in many studies, such as Kinesio taping (KT) and retro walking exercise. $\mathrm{KT}$ is a rehabilitative taping technique that providing support and stability to muscles and joints without restricting the joint's range of motion to facilitate natural healing. ${ }^{6}$ Additionally, Kinesio taping also can improve the daily living activity of the osteoarthritis patient. Kinesio taping leads to the case of degenerative arthritis in reducing pain, improving joint function, and also improving the life quality of patients. ${ }^{7}$ Retro walking exercise is another commonly used, had been proven to improve body equilibrium, knee ROM, and activate the isometric and concentric contractions, while reducing the eccentric contraction of the quadriceps. ${ }^{8}$ Further, by limiting the eccentric muscle contraction of quadriceps, it would reduce the trauma between articular cartilage that benefits in speeding up the rehabilitation of knee OA.

Although previous studies have shown that KT and retro walking exercise improved the ROM, these interventions have never compared in older people with osteoarthritis. This study aimed to evaluate the effectiveness of Kinesio taping and retro walking exercise on the knee range of motion in older people with osteoarthritis. 


\section{METHODS}

\section{Participants}

This study participant was randomly selected using random number table based on the previous study conducted in Puskesmas Kendal Karep involving 365 respondents (280 female, 85 male). There were 30 participants agreed to participate and each participant was given a written inform consent before included to this study. Of 30 participants, 14 were included into Kinesio tape and 16 into the retro walking intervention groups. The researcher would like to find further evidence regarding the difference in influence between Kinesio taping and retro walking exercise on the elderly with osteoarthritis knee risk. Retro walking exercise is also known as step backward exercise. Additionally, retro walking exercise (step backward) is rehabilitation for low extremities on osteoarthritis knee patients.

In this research, the Kinesio taping and retro walking exercise intervention in Puskesmas Kendal Karep Polehan were given three times a week on Tuesday, Thursday, and Saturday. This research employs a quantitative approach by using quasiexperimental with pretest and post-test group design, which aims to know the causality between independent and dependent variables in a certain period. Furthermore, experimental research is research done by conducting direct experiments on the tested variable. This research is conducted for 3 weeks. Additionally, Kinesio taping and retro walking exercises are given three times a week from April - May 2018. Next, the ROM is measured by using a goniometer within a month interval.

This research was calculated by statistics nonparametric using Wilcoxon signed ranked test

Table 1. The range of motion changes within 30 participants before and after Kinesio tape and retro walking exercise using Wilcoxon sign ranked test

\begin{tabular}{lcc}
\multicolumn{1}{c}{ Characteristics } & Mean rank & P-value \\
\hline Kinesio tape, degrees, $n=14$ & & \\
$\quad$ Pre-test & 0.00 & $<0.001$ \\
$\quad$ Post-test & 7.00 & \\
Retro walking exercise, degrees, $n=16$ & & \\
$\quad$ Pre-test & 7.64 & 0.019 \\
$\quad$ Post-test & 7.75 & \\
\hline
\end{tabular}

Table 2. The range of motion changes between 30 participants after Kinesio tape and retro walking exercise using Mann Whitney U test

\begin{tabular}{ccc}
\hline Characteristics & Mean & P-value \\
\hline Kinesio tape, degrees, $n=14$ & 7.8 & \\
Retro walking exercise, degrees, $n=16$ & 1.5 & 0.010 \\
\hline
\end{tabular}

in testing paired data at interval/ratio scaled, and Mann Whitney $\mathrm{U}$ test in two free groups. The conclusion was also taken by using the SPSS application to compare $P$ 's value (sig 2 tailed) with the value of $\alpha(0.05)$. As a note, $\mathrm{H} 0$ was accepted if $p$ $>0.05$, and $\mathrm{H} 1$ was accepted if the value of $p \leq 0.05$.

\section{RESULTS}

The ROM improvement before and after given Kinesio taping showed the result as follows: Kinesio tape improved the knee's ROM in the goniometer measurement from the baseline with $P<0.001$ (Table 1). The Kinesio tape gave a positive effect on the knee's range of motion in elderly with knee osteoarthritis.

The measurement using the goniometer from the pretest and post-test showed a ROM improvement after the retro walking exercise with $P=0.019$ (Table 1). There was an influence on giving retro walking exercise towards ROM improvement in elderly with knee osteoarthritis.

The measurement using goniometer towards two intervention groups showed that Kinesio tape had bigger ROM improvement than retro walking exercise with $P=0.010$ (Table 2). Kinesio tape was more beneficial for knee ROM in elderly with osteoarthritis than retro walking exercise.

\section{DISCUSSION}

Basically, Kinesio taping has different physiological effects from retro walking exercise. Kinesio taping can stimulate mechanoreceptors on the skin, causing an increase of efferent feedback to the central nervous system, causing pain reduction. ${ }^{9}$ In this study, Kinesio tape used on the skin, as the muscle and tendon are free to be contracted actively. It might pull the skin off from the underneath tissues, causing the space for blood circulation and lymphatic flow smoothly. Thus, it can reduce edema, clean the inflamed tissue, reduce pain, and faster the healing process. ${ }^{10}$ Moreover, Kinesio taping might stabilize and sustain the body's soft tissue structure (including muscle, tendon, ligament, and joints), facilitated the knee joint's correct movement, and supplement the strengthening exercise in the elderly.

In this study, the retro walking exercise showed to be effective for overcoming the knee range of motion deficits. Retro walking exercise is a part of a closed kinetic chain that is traditionally doing backward walking, making the backward push on the knees posteriorly and activating the knee's muscles, ${ }^{11}$ especially we targeted the quadriceps, hamstrings, and calf muscles. Retro walking recruited more muscle activity than 
forwarding walking, which suggested increasing joint proprioception. ${ }^{12}$ Moreover, the retro walking exercise effectively reduced knee pain because it decreased the excess adductor moment at the knee joint, which would lower the compressive forces on the medial compartment of the knee joint. ${ }^{11}$

Therefore, this research showed that Kinesio taping was more effective as it can stabilize and support the soft tissue structure; thus, the movement activation of the elderly in daily life can be facilitated. On the other hand, retro walking exercise is an exercise aimed at strengthening the muscle. Moreover, there will be no maximum effect if retro walking exercise is not conducted regularly. Hence, muscle strengthening on retro walking exercise cannot be felt directly as the effect will be felt if the exercise is done regularly.

This study has several limitations that need to explain. Firstly, this study result cannot be generalized to the population out of the study setting. This study's population has participated in another research study, which possibly had better knowledge or compliance to the intervention period. Secondly, the study participant was relatively small. The statistical power of the effects of study interventions possible change in bigger populations. Further, this study did not follow up on the participants' ROM after the intervention, which possibly changes after the study period was unknown.

\section{CONCLUSION}

Our study confirmed that Kinesio taping and retro walking interventions improved the ROM in the elderly with knee osteoarthritis. Further, the Kinesio tape seemed to give a better outcome for dealing with ROM deficit.

\section{CONFLICT OF INTEREST}

No conflict of interest to declare.

\section{FUNDING}

Not applicable

\section{AUTHOR CONTRIBUTIONS}

YPSP conceived the study design and data collection and drafted the manuscript; AY collected the data and drafted the manuscript.

\section{REFERENCES:}

1. Pai YC, Rymer WZ, Chang RW, Sharma L. Effect of age and osteoarthritis on knee proprioception. Arthritis \& Rheumatism: Official Journal of the American College of Rheumatology. 1997;40(12):2260-5.

2. Organization WH, Ageing WHO, Unit LC. WHO global report on falls prevention in older age: World Health Organization; 2008.

3. Alshami AM. Knee osteoarthritis related pain: a narrative review of diagnosis and treatment. International journal of health sciences. 2014;8(1):85.

4. Norkin CC, White DJ. Measurement of joint motion: a guide to goniometry: FA Davis; 2016.

5. Gogia PP, Braatz JH, Rose SJ, Norton BJ. Reliability and validity of goniometric measurements at the knee. Phys Ther. 1987;67(2):192-5.

6. Williams S, Whatman C, Hume PA, Sheerin K. Kinesio taping in treatment and prevention of sports injuries. Sports Med. 2012;42(2):153-64.

7. Lee K, Yi C-W, Lee S. The effects of kinesiology taping therapy on degenerative knee arthritis patients' pain, function, and joint range of motion. Journal of physical therapy science. 2016;28(1):63-6.

8. Alghadir AH, Anwer S, Sarkar B, Paul AK, Anwar D. Effect of 6-week retro or forward walking program on pain, functional disability, quadriceps muscle strength, and performance in individuals with knee osteoarthritis: a randomized controlled trial (retro-walking trial). BMC Musculoskelet Disord. 2019;20(1):159.

9. Campolo M, Babu J, Dmochowska K, Scariah S, Varughese J. A comparison of two taping techniques (kinesio and mcconnell) and their effect on anterior knee pain during functional activities. Int J Sports Phys Ther. 2013;8(2):105.

10. Li X, Zhou X, Liu H, Chen N, Liang J, Yang X, et al. Effects of elastic therapeutic taping on knee osteoarthritis: A systematic review and meta-analysis. Aging Dis. 2018;9(2):296.

11. Gondhalekar GA, Deo MV. Retrowalking as an adjunct to conventional treatment versus conventional treatment alone on pain and disability in patients with acute exacerbation of chronic knee osteoarthritis: a randomized clinical trial. N Am J Med Sci. 2013;5(2):108.

12. Yang Y-R, Yen J-G, Wang R-Y, Yen L-L, Lieu F-K. Gait outcomes after additional backward walking training in patients with stroke: a randomized controlled trial. Clin Rehabil. 2005;19(3):264-73.

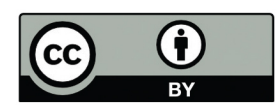

This work is licensed under a Creative Commons Attribution 